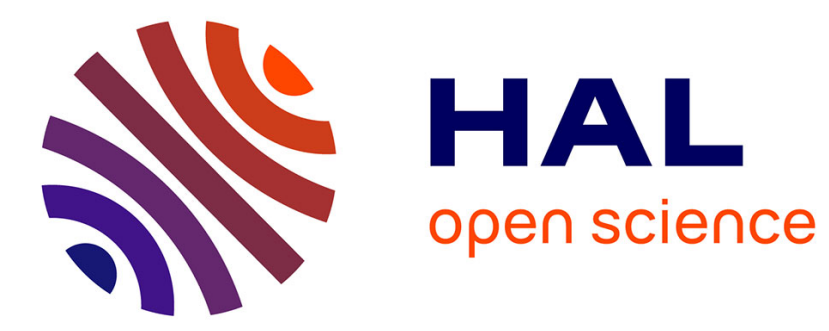

\title{
Hybrid interface: Integrating BCI in multimodal human-machine interfaces
}

Emmanuel Kalunga, Sylvain Chevallier, Olivier Rabreau, Eric Monacelli

\section{To cite this version:}

Emmanuel Kalunga, Sylvain Chevallier, Olivier Rabreau, Eric Monacelli. Hybrid interface: Integrating BCI in multimodal human-machine interfaces. IEEE/ASME International Conference on Advanced Intelligent Mechatronics, Jul 2014, Besançon, France. pp.530 - 535 10.1109/AIM.2014.6878132 . hal01352056

\section{HAL Id: hal-01352056 https://hal.science/hal-01352056}

Submitted on 5 Aug 2016

HAL is a multi-disciplinary open access archive for the deposit and dissemination of scientific research documents, whether they are published or not. The documents may come from teaching and research institutions in France or abroad, or from public or private research centers.
L'archive ouverte pluridisciplinaire HAL, est destinée au dépôt et à la diffusion de documents scientifiques de niveau recherche, publiés ou non, émanant des établissements d'enseignement et de recherche français ou étrangers, des laboratoires publics ou privés. 


\title{
Hybrid interface: Integrating BCI in Multimodal Human-Machine Interfaces
}

\author{
Emmanuel K. Kalunga ${ }^{1,2}$, Sylvain Chevallier ${ }^{1}$, Olivier Rabreau ${ }^{1}$, Eric Monacelli ${ }^{1}$
}

\begin{abstract}
In the context of assistive technologies, it is important to design systems that adapt to the user specificities, and to rely as much as possible on the residual capacities of each user. We define a new methodology in the context of assistive robotics: it is an hybrid approach where a physical interface is complemented by a Brain-Computer Interface (BCI). An implementation of such methodology is proposed, using a 3D touchless interface for continuous control and a steady-state visually evoked potential (SSVEP)-based BCI for triggering specific actions. We describe a novel algorithm for classification of SSVEP signals based on Canonical Correlation Analysis (CCA) and Support Vector Machines (SVM). Its reliability and robustness are assessed in an online setup and its results are compared to existing algorithms. Finally, an experimental evaluation of the proposed system is performed with a 3D navigation task in a Virtual Environment (VE). The system is also embedded on an assistive robotic arm exoskeleton to validate its feasibility.
\end{abstract}

\section{INTRODUCTION}

Rehabilitation and assistive technologies aim at developing solutions adapted to the subjects' disabilities. A crucial aspect is to take into account the specificities of each person and to propose technical solutions which make use of their residual motor capabilities. Hence in this work, we propose a new methodology for disabled people, using an hybrid approach where a physical interface is complemented by a Brain-Computer Interface (BCI). BCI do not rely on subjects' residual motor capabilities but current system have shown limited performances. To overcome these limitations, we design a system that allows the users to use both their brain signals and their residual motor abilities for control tasks. Our contribution are threefold : we define a new methodology for a hybrid control system, we introduce a new learning scheme for SSVEP-based BCI and we propose an implementation of the whole system in two applications of rehabilitation robotics.

BCI endows a system with communication capability through non-muscular channels, relying on brain signals [1], [2]. A recent trend focuses on the extension of BCI for healthy users [3], particularly in navigation in virtual environments and video games [4]. However the performance achieved by these systems are still far behind those of traditional assistive and HMI devices, such as joysticks. The notorious poor spatial resolution of EEG and its vulnerability

\footnotetext{
1 With Laboratoire d'Ingénierie des Systèmes de Versailles, Université de Versailles Saint-Quentin, Velizy 78140, France emmanuelkalunga.k@gmail. com

${ }^{2}$ With the Department of Electrical Engineering and the French South African Institute of Technology, Tshwane University of Technology, Pretoria 0001, South Africa
}

to artifacts [5] result in low classification accuracy and thus slow information transfer rate [2]. Also, BCIs are afflicted by a high cognitive load for the user, especially when engaged in a continuous control scheme. The last limitation is known as BCI-inefficiency or BCI illiteracy [6], [7]; a large proportion of the users (15 to $30 \%$ ) obtain poor classification results with BCI system, even with state of the art algorithms. Because of these reasons, BCIs are a possible alternative for persons with specific motor disabilities but they are not mature enough to be proposed to a larger audience.

To address these issues, two categories of approaches are found in the literature. The first, which can be regarded as a low level solution, is the refinement of signal processing techniques to enhance the EEG signal quality and pattern recognition. The second, which can be regarded as a high level adjustment, is a change in BCI paradigms. This last category is about creating new paradigms that ease the discomfort of using BCI systems and increase their performance. It has given rise to hybrid BCI approaches where two or more signals are combined in different ways to control a system. These signals can all be neurological phenomena in EEG (e.g. SSVEP and P300 [8]), or EEG and another biologic signal (e.g. EEG and EMG [9]). Another approach is to use BCI as a switch for goal selection or for performing predefined tasks that are carried on by the controlled interface to avoid a continuous control from the user [10]. We can also mention the approach of shared control between brain commands and intelligent systems where information from the environment are used to dynamically adapt the behaviour of the BCI system [11]. In each of these approaches, one of the following problem persist: either the cognitive load is still high, or the degree of freedom is very low, or the information transfer rate is low or the user's choices are predefined and thus lacks flexibility.

We propose a new methodology, where the system integrates a BCI as a complementary communication channel. This hybrid system make use of user's residual motor abilities and offers BCI as an optional choice: the user chooses when to rely on BCI and could alternate between the muscular- and brain-mediated interface at the appropriate time. Our hybrid system integrates a 3D touchless interface based on IR-sensors [12] that captures hand poses and an SSVEP-based BCI. Such an approach combines these two interfaces in a multimodal BCI-motor system that takes advantage of both the user's brain signals and her residual motor ability.

Regarding the touchless interface, our IR-based interface does not need to be held by the user, thus not requiring 
any grasping capability. It provides a 3 degrees of freedom controller. On the BCI side, we rely on SSVEP which is the response of the visual cortex to stimuli with strong frequency information [13], such as blinking stimuli. The choice of the SSVEP paradigm is motivated by the fact that it has a better signal-to-noise ratio compared to other brain activities used in BCI and it is emulated with little training as it is an inherent response of the brain [14]. We propose an approach to process SSVEP with a novel algorithm based on Canonical Correlation Analysis (CCA).

\section{STATE OF THE ART}

\section{A. Hybrid BCI Interfaces}

Combining BCI with existing Assistive Technologies (AT) has the potential of augmenting the capabilities of disabled people. In the literature, this approach is refereed to as hybrid BCI (hBCI).

The existing hBCI can be categorized according to (1) the type of signals combined and (2) how the signals are combined to achieve the desired task. According to the type of signal used, two types of hBCI are distinguished. In the first type, different brain signals (e.g. motor imagery, evoked potentials) are combined [15]-[17], while in the second a brain signal is combined with other biosignals e.g. ECG [18] or EMG [9]. The hBCI combining EMG and a brain signal is the only case where the residual muscular functionalities of the patients are used. Apart from this approach, residual muscular functionalities have been combined with BCI in a neuroprosthesis where the patient uses arm movement for reaching positions and BCI for grasping objects [19], [20].

Depending on the combination of interfaces (or control channel), several control strategies are possible. The first one is to assign one specific task per interface. Another possibility is to merge all interfaces in a weighted combination to achieve a unique task with higher accuracy. Finally, they can be used alternatively so as to allow users to smoothly switch from one interface to another depending on their performance or preference. The work of [21] provides a comprehensive review of the existing hBCI approaches and their applications. The approach proposed in this work falls under the first category of strategies. One interface is in charge of continuous control while the other is used to achieve predefined tasks (usually difficult and/or recurrent).

\section{B. SSVEP Algorithms}

Common methods for processing SSVEP are Independent Component Analysis (ICA) [22], Common Spatial Pattern (CSP) [23], and Principal Component Analysis (PCA) [24]. Most recently Canonical Correlation Analysis (CCA) [25] have been explored for signal enhancement in SSVEP. For SSVEP classification, a baseline algorithm shows good performances [26], relying only on the detection of the highest frequency component in the signal [26]. Standard classification algorithms such as LDA [11], neural networks [27], [28] or SVM [29] have also yield good results. In [29], the authors implement a code modulated-VEP BCI, a paradigm close to SSVEP, where CCA is used for the design of a spatial filter. A one-class SVM classifies the filtered EEG with high accuracy. In this work we propose a similar approach, modified and applied to regular SSVEP as described in section III.

\section{Touchless Interface}

The objective of this study is to propose an hybrid approach, relying on the residual motor capabilities combined with the SSVEP brain signals (fig. 2), to design a robust and adaptive system. We chose a 3D touchless interface as the main physical controller mainly because this device could be modified for different users, with different abilities and disabilities. The main advantage over EMG-based sensors is that touchless interface are less invasive and require no prior preparation.

In HMI, hand gestures are an active research topic [30], the main drawback of image-based approaches are the high computational cost of the existing solutions. The proposed system is meant to be embedded, working with limited resources, excluding computationally demanding algorithms. We propose to use a touchless interface based on IR-sensors, such as [31], but adapted to the motor capacities of persons affected by muscular dystrophy and neuro-degenerative disorders. Existing devices, as Leap Motion, assume a continuous and tonic activity from the user and offer no resting position.

\section{Arm Exoskeleton Control}

The control of an arm exoskeleton is presented here as a concrete application for our hybrid system. Exoskeleton for upper limb offers a useful technology for assistive [32] and rehabilitation robotics [33]. In the context of this paper, we rely on the ESTA robotic exoskeleton [34]. ESTA is designed to compensate for muscular dystrophy in the shoulder and elbow muscles occurring in several degenerative diseases, which affect the large muscles but spare the wrists and hands motor capacities.

\section{Method AND MATERIALS}

\section{A. Touchless Interface}

Our touchless interface embeds 5 IR-sensors which could set up in different spatial positions, according to the user requirements. The control system relies on a iterative $\mathrm{kNN}$ scheme to learn hand poses of each user. The details of the algorithm is provided in [12], hence only the key aspects are exposed here. Firstly, the iterative kNN scheme requires a fast calibration phase to learn the different hand poses, here seven (six for the directions and one for the resting position). The outliers and ambiguous examples are excluded from the training examples. Secondly, the algorithm continuously adapt to the received signal, labelling new examples change the set of neighbours. This algorithm is able to track the changes of the user's hand pose, providing an online adaptation to the behavioural modifications induced by tiredness. The interface is shown in Fig. 1. 


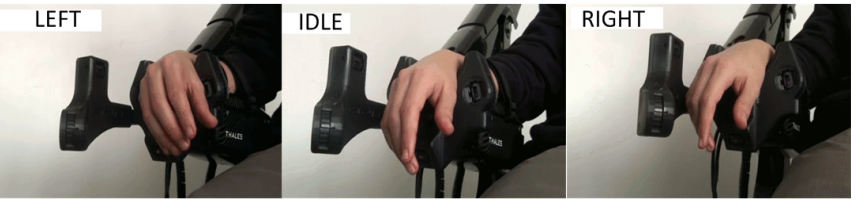

Fig. 1. The 3D touchless interface and examples of three hand poses: left, resting and right positions. The IR-sensors are in the black plastic housing on the right side of the hand and around the wrist. Another symmetrical plastic housing have been realised for left-handed users.

\section{B. SSVEP-based BCI}

The g.Mobilab+ device is used for recording EEG at $256 \mathrm{~Hz}$ on 8 channels. For SSVEP stimulation, flash stimulus technique has been chosen. To avoid limitation imposed by refresh rate of computer screens, a microcontroller is set up to flash stimuli with light emitting diodes (LED) at frequencies $F=\{13,17,21\} \mathrm{Hz}$. The device has been controlled and the LED blinking is precise up to the millisecond. The eight electrodes are placed according to the $10 / 20$ system on $\mathrm{Oz}, \mathrm{O} 1, \mathrm{O} 2, \mathrm{POz}, \mathrm{PO} 3, \mathrm{PO} 4, \mathrm{PO} 7$ and PO8. The ground was placed on $\mathrm{Fz}$ and the reference was located on the right (or left) hear mastoid.

Overlaping EEG epochs of 3 seconds are recorded every 0.5 second. Each epoch is filtered between $12 \mathrm{~Hz}$ and $45 \mathrm{~Hz}$ to discard irrelevant bands while allowing all stimulation frequencies and their first harmonics. A spatial filter is then designed based on CCA: let $X$ be the obtained EEG signal in $\mathbb{R}^{n \times m}$, where $n$ is the number of channels (8) and $m$ the sample length $(3 \times 256)$. A signal $Y$ is generated using sinusoid functions with a frequency $f \in F$ and its $k$ harmonics, i.e. $\mathrm{Y} \in \mathbb{R}^{p \times m}$ with $p=k+1$. The CCA is obtained by maximising the correlation $\rho$ between $X^{T} w_{x}$ and $Y^{T} w_{y}$ as shown in Eq. (1), where $w_{x}$ and $w_{y}$ are vectors respectively in $\mathbb{R}^{n}$ and $\mathbb{R}^{p}$.

$$
\rho=\max _{w_{x}, w_{y}} \frac{w_{x}^{T} X Y^{T} w_{y}}{\sqrt{w_{x}^{T} X X^{T} w_{x} w_{y}^{T} Y Y^{T} w_{y}}} .
$$

The filtered signal $\hat{x}$, of dimension $m$, is obtained as:

$$
\hat{x}=w_{x}^{T} X
$$

The features $\bar{X}$ provided to the classifier are defined as:

$$
\bar{X}=P_{\hat{x}}(\omega)
$$

with $\omega \in \Omega$ and $P_{\hat{x}}(\cdot)$ being the power spectral density (PSD).

Unlike the work of [25], which propose to rely on the correlation coefficient of CCA for processing SSVEP signal, in our algorithm the CCA is only applied to determine the spatial filter $w_{x}$. This produce a single signal $\hat{x}$ which is a linear combination of all the electrodes and which is expected to maximize the correlation with an hypothetically perfect neural response, that is the sinusoids of $Y$. A similar approach can be found in [29] but in a different context and using $\hat{x}$ to generate exemplars for supervised learning. Here, we propose a novel algorithm where the PSD of $\hat{x}$ is used to obtain the features of the classification process. We rely on a SVM to classify the EEG signal in four classes: the three stimuli frequency $F(13,17$ and $21 \mathrm{~Hz})$ and a reject class. We use the LIBSVM implementation with standard RBF kernels.

\section{Proposed Hybrid Interface}

The proposed hybrid approach is illustrated in Fig. 2. The user - a person with motor disabilities - is given two modalities to control the system. The first modality is an input device that takes a signal generated by users' motor action. This might be any type of device that is adapted to the subject's disability, allowing her to use her residual motor ability. This modality is used for the continuous control of the system.

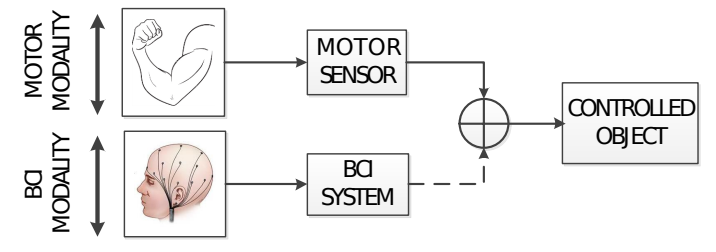

Fig. 2. Hybrid BCI system integration. The motor abilities of the user are the primary controller of the system, using adapted interfaces (here: 3D touchless interface). The brain computer interface (here: SSVEP) provides a complementary communication channel and is designed to trigger specific actions.

The second modality, which is BCI based is used to provide an additional command, giving alternative control options to the user, or a special command to activate a common and repetitive task. In this work, the continuous control is achieve with the described touchless interface and the BCI modality relies on SSVEP.

\section{Applications}

The described approach is validated in two contexts: a Virtual Environment (VE) for the navigation of a helicopter shown in Fig. 5, and an exoskeleton arm control task shown in Fig. 6. In the VE, the user is asked to reach three waypoints. Three specific locations are identified in the VE to serve as shortcuts. In previous works, locations of this nature have been used as predefined final destination [10], while we only use them as shortcuts. After reaching these locations using BCI commands, the user could reach any position using the 3D-touchless interface.

The approach with the exoskeleton arm bears some similitude with the VE navigation task. The arm is control with the 3D-touchless interface. Common arm movements performed by the user are predefined (e.g. reaching the mouth or a resting position). The $\mathrm{BCI}$ shortcut trigger the automatic arm movement to these positions.

The hybrid scheme is especially well suited for exoskeleton arm control task: as the arm is continuously controlled by the 3D touchless interface, once the user has grabbed an object (e.g. a glass of water), she will no longer be able to move her hand freely to control the touchless interface. The BCI command allows to overcome this limitation by activating predefined movements. 


\section{EXPERIMENTAL RESULTS}

This section describes the results obtained with the proposed system. Five subjects participated in the experiments. One of the subject is hemiplegic and the four others are healthy. The first section is dedicated to the assessment of our online detection of SSVEP algorithm. The next section provides the results obtained using the hybrid system for a navigation task in a virtual environment. The last section explained how the system has been implemented on an embedded system for an exoskeleton arm control task.

\section{A. Validation of our SSVEP Algorithm}

Before using the BCI subsystem in online mode, a calibration phase is needed to compute the CCA spatial filter $w_{x}$ of Eq (1) and training the SVM classifier. During the calibration phase, a sequence of trials is proposed to the user. A trial begin by an audio cue indicating which LED to focus on, or to focus on a fixation point set at an equal distance from all LEDs for the reject class. A trial last 5 seconds and there is a 3 second pause between each trial. The evaluation is conducted during a session consisting of 32 trials, with 8 trials for each frequency $f \in F$ and 8 trials for the reject class, i.e. when the subject is not focusing on any specific blinking LED. The online classification is done every 0.5 second, using a $t_{W}=3 \mathrm{~s}$ window of EEG signals. An audio feedback indicates the predicted class to the user.

Figure 3 shows the online BCI classification performances for each prediction made every 0.5 second, starting at $t=$ $t_{0}+t_{W}$, that is 3 seconds after the beginning of the trial $t_{0}$. The $y$-axis indicates the error rate for each of the five subjects. The results demonstrate that our algorithm is very robust and provides a very reliable response after $t+2 \mathrm{~s}$ with a small mean error rate for all subjects.

To further evaluate the algorithm, it is important to consider that the loss function is not uniform. If the algorithm detects a reject class instead of a specific class, the consequences are not as bad as a wrong prediction: e.g. detecting $13 \mathrm{~Hz}$ instead of $17 \mathrm{~Hz}$, as the user needs only to concentrate half a second on the chosen LED before the system make another prediction. Thus we propose the following accuracy measure, similar to a precision score. For each trial, we consider the first class prediction at time $t$ : if this is correct the accuracy is increased, if this is false the accuracy is decreased. If the prediction is the reject class, the accuracy measure is only postponed on the next time segment. Figure 4 displays the results of this measure for all subjects. The accuracy is above $70 \%$ for almost all subjects and it can be seen that the algorithm provides almost immediately the correct answer.

At last, we compare our algorithm with classical SSVEP approaches in Table I, using an offline evaluation for each subject. The baseline is a comparison with a SVM using the PSD of the EEG signal, that is without applying the CCA spatial filter. A classical methodology is to rely on ICA to extract the main components of the signal and to provide these components to the SVM classifier. Table I shows that our algorithm yields the best results.

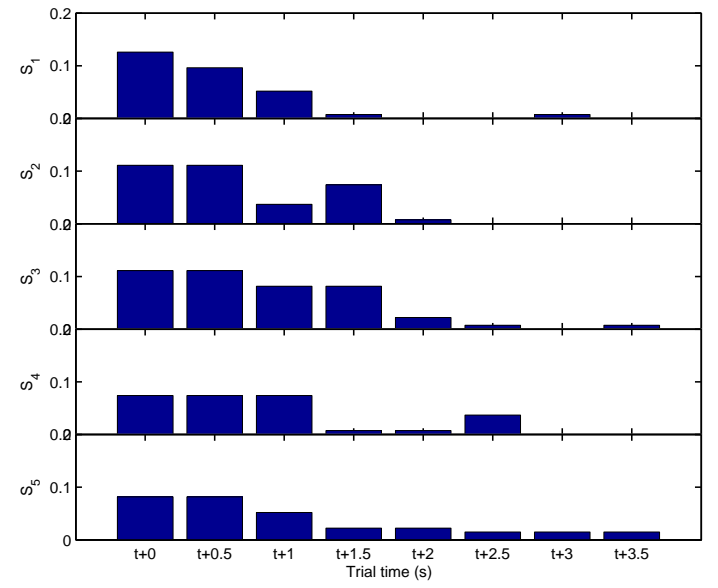

Fig. 3. Evaluation of the online performances of our BCI algorithm. The error rates for all five subjects are indicated as a function of time, with $t+0$ indicating the first prediction made (after $t_{W}=3 \mathrm{~s}$ ). The error rates are averaged on all classes.

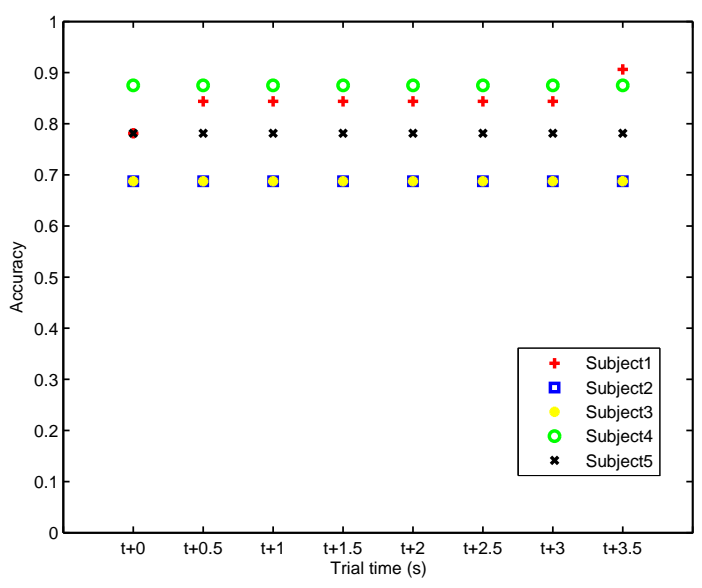

Fig. 4. Assessment of the accuracy of classification depending on the time of the prediction. On $\mathrm{x}$-axis, $t+0$ indicates the first prediction made 3 seconds after the start of a trial. The results are averaged on all trials for each subjects. Subject 1 is the only one to present a slight increase of the classification accuracy. For all other subjects the algorithm proposes a correct answer as the first prediction.

TABLE I

COMPARISON WITH OTHER ALGORITHMS

\begin{tabular}{lrrrrr}
\hline & Subject1 & Subject2 & Subject3 & Subject4 & Subject5 \\
\hline Baseline & 81.3 & 88.3 & 80.0 & 75.0 & 79.2 \\
\hline ICA & $\mathbf{1 0 0}$ & 88.3 & 91.7 & $\mathbf{9 3 . 3}$ & 95.0 \\
\hline CCA & $\mathbf{1 0 0}$ & $\mathbf{1 0 0}$ & $\mathbf{9 7 . 5}$ & $\mathbf{9 3 . 3}$ & $\mathbf{9 6 . 7}$ \\
\hline
\end{tabular}

\section{B. Experiments in Virtual Environment}

For the navigation task in the virtual environment, the assessment is based on the time spent and the distance travelled during the experiment for 4 subjects. These results are shown in Table II. The time is indicated in seconds and the distance in metric units. Each subject has performed 3 experiments: in the first experimental condition, the subject should rely only on the 3D touchless interface ('None' in Table II). In the 


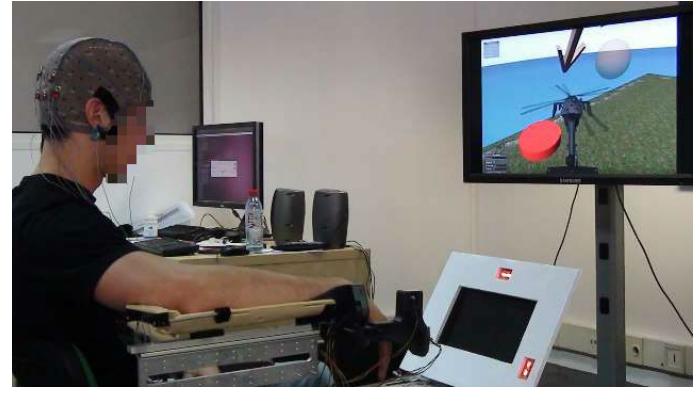

Fig. 5. Experiment in the virtual environment. Here the subject is using the 3D touchless interface with his right hand and the SSVEP LEDs are put in front of him. The screen displays an helicopter in the virtual environment. The subject should pass through all waypoints, materialised by red (or grey) disks on the screen. When the subject triggers a shortcut, the helicopter is moved to a location materialized by the transparent ball.

second one, shortcuts are available and are triggered by the BCI subsystem (BCI-S). In the last experimental condition, the subject could trigger a shortcut using a keyboard (KB$\mathrm{S})$. The fourth subject is hemiplegic and she could not use the keyboard with her spare hand. Thus, her results do not include the last experimental condition.

In Table II, next to the BCI and keyboard shortcut, a percentage indicates the relative improvement compared to the reference experiment (without shortcut). It could be seen that distance covered is almost equivalent with BCI shortcuts and keyboard shortcuts, which is the expected results as users have activated the shortcut each time it was possible. When the shortcuts are activated by the $\mathrm{BCI}$, the task is slower than when using the keyboard. This effect is mainly caused because the subject need to focus at least 3 seconds on a blinking LED before triggering the shortcut.

TABLE II

DISTANCE COVERED AND DURATION OF EXPERIMENTS, WITHOUT SHORTCUTS (NONE), WITH BCI-ACTIVATED SHORTCUT (BCI-S) AND WITH KEYBOARD-ACTIVATED SHORTCUT (KB-S).

\begin{tabular}{ccrrr}
\hline & & None & BCI-S (inc. \%). & KB-S (inc. \%) \\
\hline \multirow{2}{*}{ Subject 1 } & Time & 108.9 & $68.3(37.3 \%)$ & $53.56(50.8 \%)$ \\
\cline { 2 - 5 } & Distance & 1367.7 & $538.2(60.6 \%)$ & $535.0(60.9 \%)$ \\
\hline \multirow{2}{*}{ Subject 2 } & Time & 99.2 & $74.7(24.7 \%)$ & $50.5(49.1 \%)$ \\
\cline { 2 - 5 } & Distance & 1469.4 & $529.1(64.0 \%)$ & $549.0(62.6 \%)$ \\
\hline \multirow{2}{*}{ Subject 3 } & Time & 105.5 & $63.4(39.9 \%)$ & $50.4(52.2 \%)$ \\
\cline { 2 - 5 } & Distance & 1447.3 & $627.6(56.6 \%)$ & $542.1(62.5 \%)$ \\
\hline \multirow{2}{*}{$\begin{array}{c}\text { Subject 4 } \\
\text { (hemiplegic) }\end{array}$} & Time & 125.6 & $70.4(43.9 \%)$ & \\
\cline { 2 - 5 } & Distance & 1490.8 & $598.9(59.8 \%)$ & \\
\hline
\end{tabular}

\section{Application to Exoskeleton Arm Control}

The proposed system have been applied to the ESTA exoskeleton arm control. This assistive device is designed to compensate shoulder and elbow deficiencies occurring in degenerative diseases. Fig. 6 shows the 3D touchless interface equipped at the extremity of the ESTA arm. LEDs are placed on the left side, a close-up picture is displayed on the top left part of the figure.

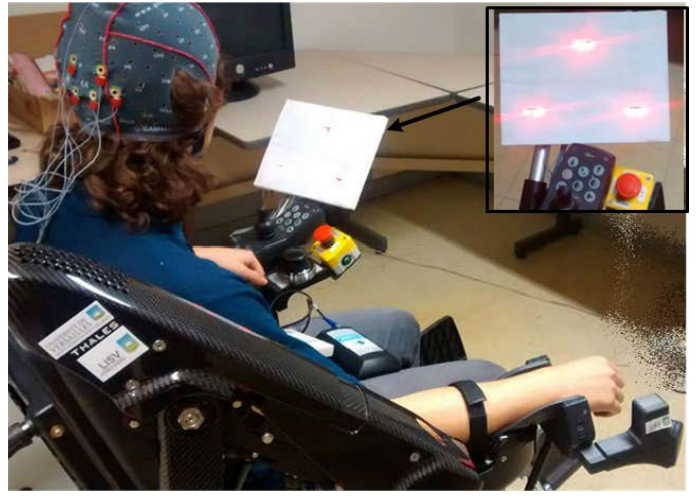

Fig. 6. The ESTA arm exoskeleton with the proposed system: a 3Dtouchless interface and the SSVEP-based BCI.

The subject controls the exoskeleton arm with the touchless interface and the BCI shortcuts allows to reach predefined positions, such as a resting or a close-to-mouth positions. In the case of the hemiplegic subject (who can not use her left arm and hand), the BCI subsystem is the only possibility to control the exoskeleton with an object in hand. This example illustrates the complementary aspect of the two interfaces, the physical and the brain one.

\section{CONCLUSION}

We introduce a new methodology for designing hybrid systems, using both a physical interface and a BCI. The main goal of these hybrid systems is to assist people with motor disabilities or muscular diseases, by proposing a system which make use of their residual abilities. The BCI is integrated in the system as a secondary interface, which is used to trigger specific behavior or predefined actions.

A first contribution is to propose an implementation of such a system using a 3D touchless interface and a SSVEPbased BCI. This implementation gather the two interfaces in a multimodal system which benefits from both the brain and motor signals. Our second contribution is to describe a novel algorithm for processing SSVEP-based EEG signals, with stable results, even when computed in an online setup. This algorithm is compared to other existing solutions and an experimental assessment of its validity is conducted.

The full system is evaluated on a 3D navigation task in virtual environment. The results demonstrate that the system is functional and could be used to assist people in various contexts. We also implement our system to control the ESTA arm exoskeleton: the system is functional and could be adapted for controlling other assistive devices.

Future works is focused on adding adaptive properties to the system, enabling the system to adapt to the behavioral variations of the user.

\section{REFERENCES}

[1] G. Pfurtscheller, D. Flotzinger, and J. Kalcher, "Brain-Computer interface - a new communication device for handicapped persons," Journal of Microcomputer Applications, vol. 16, no. 3, pp. 293-299, Jul. 1993. 
[2] J. Wolpaw, N. Birbaumer, D. J. McFarland, G. Pfurtscheller, and T. M. Vaughan, "Brain-computer interfaces for communication and control," Clinical Neurophysiology, vol. 113, no. 6, pp. 767-791, Jun. 2002.

[3] M. Tangermann, K.-R. Müller, A. Aertsen, N. Birbaumer, C. Braun, C. Brunner, R. Leeb, C. Mehring, K. J. Miller, G. Mueller-Putz, G. Nolte, G. Pfurtscheller, H. Preissl, G. Schalk, A. Schlögl, C. Vidaurre, S. Waldert, and B. Blankertz, "Review of the BCI Competition IV," Frontiers in Neuroscience, vol. 6, no. 55, 2012.

[4] Y. Renard, F. Lotte, G. Gibert, M. Congedo, E. Maby, V. Delannoy, O. Bertrand, and A. Lécuyer, "OpenViBE: An Open-Source software platform to design, test, and use Brain-Computer interfaces in real and virtual environments," Presence: Teleoperators and Virtual Environments, vol. 19, no. 1, pp. 35-53, 2010.

[5] E. Niedermeyer and F. Lopes da Silva, Electroencephalography: Basic Principles, Clinical Applications, and Related Fields, 5th ed. Lippincott Williams \& Wilkins, 2004.

[6] C. Vidaurre and B. Blankertz, "Towards a cure for BCI illiteracy." Brain topography, vol. 23, no. 2, pp. 194-198, 2010.

[7] E. M. Hammer, S. Halder, B. Blankertz, C. Sannelli, T. Dickhaus, S. Kleih, K.-R. Müller, and A. Kübler, "Psychological predictors of SMR-BCI performance," Biological Psychology, vol. 89, no. 1, pp. 80-86, 2012

[8] E. Yin, Z. Zhou, J. Jiang, F. Chen, Y. Liu, and D. Hu, "A novel hybrid BCI speller based on the incorporation of SSVEP into the p300 paradigm," Journal of Neural Engineering, vol. 10, no. 2, 2013.

[9] R. Leeb, H. Sagha, R. Chavarriaga, and J. del R Millan, "Multimodal Fusion of Muscle and Brain Signals for a Hybrid-BCI," Engineering in Medicine and Biology Society (EMBC), 2010 Annual International Conference of the IEEE, pp. 4343-4346, 2010

[10] F. Lotte, A. Van Langhenhove, F. Lamarche, T. Ernest, Y. Renard, B. Arnaldi, and A. Lécuyer, "Exploring large virtual environments by thoughts using a brain-computer interface based on motor imagery and high-level commands," Presence: teleoperators and virtual environments, vol. 19, no. 1, pp. 54-70, 2010.

[11] J. Legeny, R. Viciana-Abad, and A. Lécuyer, "Toward contextual ssvep-based bci controller: Smart activation of stimuli and control weighting," Computational Intelligence and AI in Games, IEEE Transactions on, vol. 5, no. 2, pp. 111-116, June 2013.

[12] H. Martin, S. Chevallier, and E. Monacelli, "Fast calibration of hand movement-based interface for arm exoskeleton control," in European Symposium on Artificial Neural Networks (ESANN), 2012, pp. 573578.

[13] A. Capilla, P. Pazo-Alvarez, A. Darriba, P. Campo, and J. Gross, "Steady-State Visual Evoked Potentials Can Be Explained by Temporal Superposition of Transient Event-Related Responses," PLoS ONE, vol. 6, no. 1, p. e14543, 2011.

[14] D. Zhu, J. Bieger, G. G. Molina, and R. M. Aarts, "A survey of stimulation methods used in SSVEP-based BCIs," Intell. Neuroscience, vol. 2010, pp. 1-12, 2010.

[15] P. Ferrez and J. del R. Millan, "Error-Related EEG Potentials Generated During Simulated Brain-Computer Interaction," Biomedical Engineering, IEEE Transactions on, vol. 55, no. 3, pp. 923-929, 2008.

[16] B. Z. Allison, C. Brunner, V. Kaiser, G. R. Muller-Putz, C. Neuper, and G. Pfurtscheller, "Toward a hybrid brain computer interface based on imagined movement and visual attention," Journal of Neural Engineering, vol. 7, no. 2, p. 026007, 2010.

[17] A. Finke, A. Knoblauch, H. Koesling, and H. Ritter, "A hybrid brain interface for a humanoid robot assistant," Engineering in Medicine and Biology Society,EMBC, 2011 Annual International Conference of the IEEE, pp. 7421-7424, 2011.

[18] R. Scherer, G. R. Müller-Putz, and G. Pfurtscheller, "Self-initiation of EEG-based brain-computer communication using the heart rate response," Journal of Neural Engineering, vol. 4, no. 4, p. L23, 2007.

[19] J. Millan, F. Galan, D. Vanhooydonck, E. Lew, J. Philips, and M. Nuttin, "Asynchronous non-invasive brain-actuated control of an intelligent wheelchair," Engineering in Medicine and Biology Society, 2009. EMBC 2009. Annual International Conference of the IEEE, pp 3361-3364, 2009

[20] J. D. R. Millan, F. Renkens, J. Mourino, and W. Gerstner, "Noninvasive brain-actuated control of a mobile robot by human EEG," IEEE Transactions on Biomedical Engineering, vol. 51, pp. 1026-1033, 2004.

[21] J. d. R. Millán, R. Rupp, G. Mueller-Putz, R. Murray-Smith, C. Giugliemma, M. Tangermann, C. Vidaurre, F. Cincotti, A. Kubler, R. Leeb, C. Neuper, K. R. Mueller, and D. Mattia, "Combining Brain-
Computer Interfaces and Assistive Technologies: State-of-the-Art and Challenges," Frontiers in Neuroscience, vol. 4, no. 161, 2010.

[22] Y. Wang, Z. Zhang, X. Gao, and S. Gao, "Lead selection for SSVEPbased brain-computer interface," Engineering in Medicine and Biology Society, 2004. IEMBS '04. 26th Annual International Conference of the IEEE, vol. 2, pp. 4507-4510, 2004.

[23] G. Pires, U. Nunes, and M. Castelo-Branco, "Statistical spatial filtering for a P300-based BCI: Tests in able-bodied, and patients with cerebral palsy and amyotrophic lateral sclerosis," Journal of Neuroscience Methods, vol. 195, no. 2, pp. 270-281, 2011.

[24] R. Ren, G. Bin, and X. Gao, "Idle state detection in ssvep-based braincomputer interfaces," Bioinformatics and Biomedical Engineering, 2008. ICBBE 2008. The 2nd International Conference on, pp. 2012 2015, May 2008.

[25] Z. Lin, C. Zhang, W. Wu, and X. Gao, "Frequency Recognition Based on Canonical Correlation Analysis for SSVEP-Based BCIs,' Biomedical Engineering, IEEE Transactions on, vol. 53, no. 12, pp. 2610-2614, 2006

[26] M. Movahedi, A. Mehdizadeh, and A. Alipour, "Development of a brain computer interface (bci) speller system based on ssvep signals," Journal of Biomedical Physics and Engineering, vol. 3, no. 3 Sep, 2013.

[27] K. Lao, C. Wong, F. Wan, P. Mak, P. Mak, and M. Vai, "Canonical correlation analysis neural network for Steady-State visual evoked potentials based Brain-Computer interfaces," in Advances in Neural Networks - ISNN 2013, ser. Lecture Notes in Computer Science, C. Guo, Z.-G. Hou, and Z. Zeng, Eds. Springer, 2013, vol. 7952, pp. 276-283.

[28] H. Cecotti, "A time-frequency convolutional neural network for the offline classification of steady-state visual evoked potential responses,' Pattern Recognition Letters, vol. 32, no. 8, pp. 1145 - 1153, 2011.

[29] M. Spüler, W. Rosenstiel, and M. Bogdan, "One class SVM and canonical correlation analysis increase performance in a c-VEP based brain-computer interface (BCi)," in Proceedings of 20th European Symposium on Artificial Neural Networks (ESANN 2012), Bruges, Belgium, vol. 4, 2012, pp. 103-108.

[30] A. Chaudhary, J. Raheja, K. Das, and S. Raheja, "A survey on hand gesture recognition in context of soft computing advanced computing," in Communications in Computer and Information Science, 2011, vol 133, pp. 46-55.

[31] D. Ryu, D. Um, P. Tanofsky, D. Koh, Y. Ryu, and S. Kang, "T-less: A novel touchless human-machine interface based on infrared proximity sensing," in Intelligent Robots and Systems (IROS), Oct. 2010, pp. 5220-5225.

[32] B. Dellon and Y. Matsuoka, "Prosthetics, exoskeletons, and rehabilitation [grand challenges of robotics]," Robotics \& Automation Magazine, IEEE, vol. 14, no. 1, pp. 30-34, 2007.

[33] E. Rocon and J. Pons, "Introduction: Exoskeletons in rehabilitation robotics," in Springer Tracts in Advanced Robotics, 2011, vol. 69, pp. $1-20$.

[34] M. Baklouti, P. A. Guyot, E. Monacelli, and S. Couvet, "Force controlled upper-limb powered exoskeleton for rehabilitation," in Intelligent Robots and Systems (IROS), 2008, p. 4202. 